\title{
Analysis of the mechanism of Aldo-keto reductase dependent cis-platin resistance in HepG2 based on transcriptomic and NADH metabolic analysis
}

1 Tingting Sun ${ }^{1}$, Xue Sun ${ }^{1}$, Xin Wang ${ }^{1}$, Rui Guo ${ }^{1}$, Yuanhua Yu ${ }^{1 *}$, Le Gao ${ }^{*}$

$2{ }^{1}$ Changchun University of Science and Technology, Changchun, 130022, P.R. China.

3 * Correspondence:

4 Corresponding Author

5 Yuanhua $\mathrm{Yu}$

6 E-mail: yuyuanhua8888@126.com

7 Le Gao

8 E-mail: legaooagel@163.com

9 Keywords : Aldo-keto oxidoreductase $(\mathrm{AKR})_{1}$, RNA sequencing, NAD(P)H-dependent oxidoreductases 3 , Cis-platin 4 , HepG2 ${ }_{5}$.

\section{Abstract}

Aldo-keto oxidoreductase (AKR) inhibitors could reverse several cancer cells' resistance to Cis-platin, but their role in resistance remains unclear. Our RNA-seq results showed de novo NAD biosynthesis-related genes, and $\mathrm{NAD}(\mathrm{P}) \mathrm{H}$-dependent oxidoreductases were significantly upregulated in Cis-platin-resistant HepG2 hepatic cancer cells (HepG2-RC cells) compared with HepG2 cells. Knockdown of AKR1Cs could increase Cis-platin sensitivity in HepG2-RC cells about two-fold. Interestingly, the AKR1C inhibitor meclofenamic acid could increase Cis-platin sensitivity of HepG2-RC cells about eight-fold, indicating that knockdown of AKR1Cs only partially reversed the resistance. Meanwhile, the amount of total NAD and the ratio of $\mathrm{NADH} / \mathrm{NAD}+$ were increased in HepG2-RC cells compared with HepG2 cells. The increased NADH could be explained as a directly operating antioxidant to scavenge radicals induced by Cis-platin. We report here that NADH, which is produced by $\mathrm{NAD}(\mathrm{P}) \mathrm{H}$-dependent oxidoreductases, plays a key role in the AKR-associated Cis-platin resistance of HepG2 hepatic cancer cells.

\section{Introduction}

Aldo-keto reductases (AKRs) are $\mathrm{NAD}(\mathrm{P}) \mathrm{H}$-dependent oxidoreductases, which reduce carbonyl substrates with $\mathrm{NAD}(\mathrm{P}) \mathrm{H}$ and are present in all three domains of life. Human AKRs are important in metabolic pathways such as steroid biosynthesis, alcohol oxidation, and xenobiotic elimination[1]. Some AKRs, such as AKR1B10[2] and AKR1Cs[3,4], are correlated with carcinogenesis. AKR1Cs have also been related to Cis-platin resistance in gastric carcinoma TSGH-S3 cells [5] and metastatic bladder cancer cells[6]. 
Cis-platin is a front-line chemotherapy agent for cancer treatment[7]. The AKR1C inhibitor flufenamic acid was found useful to reverse Cis-platin resistance of bladder cancer[6]. It was speculated that AKR1Cs could reduce some cytotoxic lipid peroxidative products from aldehydes[5]. Hepatic cancer cells with high expression levels of AKRs usually show a rather high tolerance to Cis-platin treatment. However, the target molecule of AKRs remains unknown. Here we report that NADH, a product of Aldo-keto oxidation-reduction, plays a key role in the Cis-platin resistance of HepG2 hepatic cancer cells.

\section{Materials and Methods}

\subsection{Cell culture}

Human hepatic HL-7702 cells and the hepatic cancer cell lines HepG2 and its Cis-platin-resistant strain HepG2-RC were purchased from Fusheng Biotechnology (Shanghai, China). HL-7702 cells were cultured at 5\% CO2 in RPMI-1640 medium. HepG2 cells were cultured at 5\% CO2 in DMEM medium. HepG2-RC cells were cultured at 5\% CO2 in MEME medium with increasing Cis-platin concentration until $50 \%$ of cells died.

\subsection{Quantitative real-time polymerase chain reaction}

Total RNA isolation and first-strand cDNA synthesis from HL-7702, HepG2, and HepG2-RC cells were performed by a SuperReal Kit (Tiangen, Beijing, China). The primer sequences are listed in Table S1. Quantitative real-time polymerase chain reaction (qRT-PCR) was performed using ABI7900HTFast (Thermofisher, Waltham, MA). The data were normalized to the $\beta$-actin expression level and are expressed as the fold change relative to control $\left(2^{-\Delta \Delta \mathrm{Ct}}\right)$.

\subsection{Western blot}

Cells were washed twice with cold PBS and lysed in a buffer containing 0.5\% NP-40, $10 \mathrm{mM}$ Tris-HCl (pH 7.4), 150 mM NaCl, 1 mM EDTA, 50 mM NaF, 1 mM PMSF, and $1 \mathrm{mM} \mathrm{Na} 3 \mathrm{VO} 4$, and the lysate was clarified by centrifugation at $15,000 \mathrm{rpm}$ for 10 min. The supernatants were then subjected to $8 \%-12 \%$ SDS-PAGE. Separated proteins were transferred to polyvinylidene difluoride membranes and blocked using Tris-buffered saline containing Tween-20 (TBS-T) with 5\% skim milk at room temperature for $1 \mathrm{~h}$. Membranes were incubated with the following primary antibodies: anti-AKR1C1 (Abnova, Taiwan, China), anti-AKR1C2 (Abcam, Cambridge, UK), anti-AKR1C3 (Abcam, Cambridge, UK), and AKR1C4 (Abnova, Taiwan, China) at $4^{\circ} \mathrm{C}$ overnight. Membranes were washed with TBS-T, followed by incubation with responding secondary antibodies. The membranes were washed three times in TBS-T, and the signal was developed using ECL (GE Healthcare, Little Chalfont, UK), followed by detection using an AzureC600 detection system (Azure Biosystems, Dublin, CA).

\subsection{Measuring IC50 of AKR1C inhibitors}


73

74

75

76

77

78

79

80

81

82

83

84

85

86

87

88

89

90

91

92

93

94

95

96

97

98

99

100

101

102

103

104

105

106

107

108

109

110

The activity of AKR1C3 was estimated by measuring the OD340 (NADH) during the conversion of glyceraldehyde to glycerol by the Vallee-Hoch method. Three inhibitors, medroxyprogesterone acetate (MPA), meclofenamic acid (MCFLA), and methyliasmonate (MLS), were applied to inhibit AKR1C3 activity. The IC50 of the inhibitors was calculated from the plots of AKR1C3 activity vs concentration of inhibitors. Each sample was measured in triplicate.

\subsection{Reverse Cis-platin resistance}

MPA, MCFLA, and MLS were applied to reverse Cis-platin resistance of HepG2-RC. The inhibitors at given concentrations (MPA: $0.31 \mathrm{mM}$, MCFLA: $0.12 \mathrm{mM}$, MLS: $0.13 \mathrm{mM}$ ) were co-incubated with HepG2-RC cells under a gradient concentration of Cis-platin. The Cis-platin IC50 of each inhibitor-treated HepG2-RC sample was then calculated from the MTT assay.

For the knockdown of AKR1Cs in HepG2-RC cells. Small interfering RNAs (siRNAs) targeting human AKR1C1-4 were synthesized by Qiagen (Valencia, CA). HepG2-RC cells $(5 \times 106$ cells/well) were transfected with $50 \mathrm{nM}$ of si-AKR1Cs or si-scramble as control (sequences are provided in Table S1) using HiPerfect transfection reagent (Qiagen, Valencia, CA). After 24 or $48 \mathrm{~h}$ transfection, cells were subjected to RT-PCR and immunoblotting to identify whether AKR1Cs were knocked down. The HepG2-RC cells in which knockdown of AKR1Cs was successful then underwent a Cis-platin IC50 test as described above.

\subsection{RNA-sequencing and data analysis}

Approximately 106 HepG2 and HepG2-RC cells were frozen on dry ice. RNA extraction, library preparation, RNA-seq, and bioinformatics analysis were performed at BGI (Shenzhen, China). Each set of cell samples was sequenced in three independent experiments. Image analysis, base-calling, and filtering based on fluorescence purity and output of filtered sequencing files were performed through the Illumina analysis pipeline.

The obtained raw reads of HepG2 and HepG2-RC cells were preprocessed by removing reads containing adapter sequences, reads containing poly-N, and low-quality reads. Q20 and Q30 were calculated. All six runs of HepG2 and HepG2-RC samples showed that at least $96 \%$ of reads was Q20, and at least $87 \%$ was Q30. All downstream analyses were based on high-quality clean reads. Gene function was annotated based on Gene Ontology (GO) and the Kyoto Encyclopedia of Genes and Genomes (KEGG). Genes with $\log 2$ (fold change) $>1$ and FPKM $>0.1$ were selected as upregulated genes. Genes with $\log 2$ (fold change) $<-1$ and FPKM $>0.1$ were selected as downregulated genes. Finally, 1486 upregulated genes and 270 downregulated genes were identified.

\subsection{Assay of NADH and NAD+ content}


The amounts of NADH and NAD+ were measured by NAD+/NADH Assay Kit with WST-8 (Beyotime, Shanghai, China). Approximately 106 HL-7702, HepG2, and HepG2-RC cells were collected for NAD+ or NADH extraction. Each sample was divided into two equal parts. One part was used for measuring the total amount of $\mathrm{NAD}(\mathrm{NAD}++\mathrm{NADH})$, and the other part was used for measuring the amount of $\mathrm{NADH}$ after $60^{\circ} \mathrm{C}$ heat treatment. In the assay, NAD+ was first converted to NADH by adding alcohol dehydrogenase and ethanol. NADH then reduced WST-8 to formazan, and the amount of NADH could be measured by monitoring the OD450 value. The ratio of $\mathrm{NAD}+\mathrm{NADH}$ was calculated by the formula ratio $=\left([N A D]_{\text {total }}-\right.$ $[N A D H]) /([N A D H])$. Each sample was measured in three independent experiments.

\subsection{Docking experiment}

A protein docking model of AKR1C3 and its inhibitors was simulated by AutoDock 4.2.6 (The Scripps Research Institute, San Diego, CA). The crystal structure of AKR1C3 was derived from the RCSB protein data bank (ID: 4DBW). Ligands were initially drawn using ChemBioDraw Ultra 13.0 (PerkinElmer, Waltham, MA), and energy minimization was performed with ChemBio3D Ultra 13.0 using the MMFF94 force field (PerkinElmer, Waltham, CA). Optimized ligand candidates were saved in PDBQT format. The dimensions of the grid box were set at 110, 110, $85(\mathrm{x}, \mathrm{y}, \mathrm{z})$, and the center of the box was placed on Tyr-55 in the A-chain. MPA, MCFLA, and MLS were designed and docked onto the AKR1C3 model with or without NADP+, and their binding energy was estimated from docking models.

\section{Results}

\subsection{AKR1C3 was upregulated in HepG2-RC cells compared with HepG2 cells}

To investigate the differential expression of AKR1Cs in HepG2 and HepG2-RC cells, we evaluated their mRNA and protein levels. According to the qRT-PCR results (Table 1), the mRNA levels of all AKR1C isoenzymes were higher in HepG2 cells compared with human hepatic HL-7702 cells. However, only AKR1C3 was upregulated in HepG2-RC cells compared with HepG2 cells ( $\sim 50$-fold), while the other isoenzymes showed decreased levels in HepG2-RC cells.

According to our Western blot results (Fig.1), AKR1C1, AKR1C2, and AKR1C4 levels were almost equal between HepG2 and HepG2-RC cells (1.2-fold differences). AKR1C3 levels in HepG2-RC cells were as much as 1.5-fold higher than in HepG2 cells, which was consistent with our qRT-PCR results.

\subsection{AKR1C inhibitors could reverse Cis-platin resistance of HepG2-RC cells}

Three AKR1C inhibitors, MPA, MCFLA, and MLS, were applied to reverse Cis-platin resistance in HepG2-RC cells. First, the IC50 value of each AKR1C3 inhibitor was determined (Fig. 2A). MPA showed the lowest IC50 value $(2.1 \mu \mathrm{M})$. The IC50 values of the other inhibitors were $3.3 \mu \mathrm{M}$ (MCFLA) and $16.3 \mu \mathrm{M}$ (MLS). However, MCFLA caused the strongest increase in Cis-platin sensitivity ( $~ 8$-fold). MPA and MLS 
150

increased Cis-platin sensitivity almost 2.5 -fold and approximately 1.5 -fold, respectively (Fig. 2B).

\subsection{SiRNA of AKR1Cs could partially reverse resistance of HepG2-RC cells}

Since AKR1C inhibitors could reverse Cis-platin resistance of HepG2-RC cells, RNAi knockdown experiments of all AKR1Cs were performed. Western blot results showed that $\mathrm{AKR} 1 \mathrm{C} 1$ and $\mathrm{AKR} 1 \mathrm{C} 3$ protein levels were strongly reduced, while these remained unchanged in control-siRNA HepG2-RC cells (Fig. 2C). These observations confirm that AKR1C1 and AKR1C3 were successfully knocked down in HepG2-RC cells.

Cis-platin resistance reversal experiments showed that the AKR1C knockdown HepG2-RC cells could tolerate two-fold lower Cis-platin concentrations than control HepG2-RC cells (Fig. 2D). The effects of AKR1C knockdown on Cis-platin resistance were equivalent to the effects of MPA ( 2.5-fold reversal), but were much weaker than the effects of MCFLA ( $~ 8$-fold reversal), indicating that knockdown of AKR1Cs could partially reverse Cis-platin resistance of HepG2-RC cells.

\subsection{Most NAD(P)H-dependent reductase/oxidases were upregulated in HepG2-RC cells}

According to our RNA-seq results, AKR1C levels were not greatly changed in HepG2-RC cells ( $\log 2(\mathrm{HEPG} 2-\mathrm{RC} / \mathrm{HEPG} 2)<2$; Table 2 ), and only AKR1C3 showed a slight increase, while the other three showed a slight decrease. This tendency was consistent with the above qRT-PCR results. Among the 16 human AKR enzymes, four (AKR1B10, AKR1B15, AKR1D1, and AKR1B1) were upregulated about four-fold, while two (AKR1E2 and AKR1C4) were downregulated about twofold; the remaining nine enzymes showed almost no change.

Moreover, many other NAD(P)H-dependent reductase/oxidases were upregulated fourto eight-fold (Table 2). The strongest upregulation was observed for RFTN1 $(\log 2($ HEPG2-RC/HEPG2 $)=6.55)$, which encodes raftlin, which bears NAD $(\mathrm{P}) \mathrm{H}$ cytochrome-b5 reductase activity. Comparing RNA-seq results from HepG2 and HepG2-RC cells, 63 NAD(P)H-dependent reductase/oxidases were upregulated in HepG2-RC cells at least twofold, while only 23 were downregulated at least/approximately twofold. Moreover, 23 of those 63 upregulated genes had at least four-fold higher transcription in HepG2-RC cells compared with HepG2 cells, while only two of those 23 downregulated genes showed a reduction to less than $25 \%$ of HepG2 levels. In other words, even though no NAD(P)H-dependent reductase/oxidases were present in the top 10 upregulated genes (Tables S2 and S3), they were generally upregulated.

\subsection{Ratio of NADH/NAD+ in HepG2-RC cells was higher than in HepG2 cells}

As shown in Fig. 3, the amount of total NAD in HepG2 and HepG2-RC cells was approximately four-fold higher than in HL-7702 cells. Interestingly, the ratio of 
$\mathrm{NADH} / \mathrm{NAD}+$ in HepG2-RC cells was almost seven-fold higher than in HepG2 or HL-7702 cells. These results could be explained by the RNA-seq results that de novo NAD biosynthesis-related genes were upregulated in HepG2-RC cells (Table 3). Especially, TDO2, encoding tryptophan dioxygenase; KYNU, encoding kynureninase; and NMNAT2, encoding nicotinamide nucleotide adenylyl transferase, showed at least four-fold and at most 32-fold higher mRNA levels in HepG2-RC than in HepG2 cells. Moreover, almost all genes involved in NAD degradation did not show altered mRNA levels, except the ART family. All ART family genes were upregulated in HepG2-RC compared with HepG2; in particular, ART1 mRNA levels in HepG2-RC cells increased 32-fold compared with HepG2 cells. It has been reported that ART1 overexpression is closely related to some kinds of cancer[8-10]. In summary, the de novo NAD biosynthesis pathway was upregulated in HepG2-RC, and total NAD levels were increased in HepG2 and HepG2-RC cells compared with HL-7702 cells.

\subsection{AKR1C inhibitors could bind with AKR1C3 at different locations, as predicted by molecular docking stimulations}

Docking simulations with an NADP+-bound AKR1C3 model (Fig. 4A-C) showed that the highest affinity docking inhibitor was MPA, with the lowest binding free energy $(\Delta \mathrm{G}=-11.54 \mathrm{kcal} / \mathrm{mol})$. MCFLA showed the second highest affinity, with $\Delta \mathrm{G}=$ $-7.06 \mathrm{kcal} / \mathrm{mol}$, and MLS showed the lowest affinity $(-6.47 \mathrm{kcal} / \mathrm{mol})$. These results are consistent with the IC50 values of the inhibitors on AKR1C3, with MPA having the lowest and MLS having the highest IC50 value (Fig. 2A).

If NADP+ was removed from the AKR1C3 structure and only inhibitors were docked with AKR1C3 apoenzyme, the order did not change, but we could see considerable differences in their inhibitor binding location (Fig. 4A-C). MCFLA and MLS occupied the oxygen site composed by Tyr-55, His-117, and NADP+, while MPA was tightly bound to the steroid-binding site. In the NADP+-bound AKR1C3 model, the distance between the nitrogen atom of the nicotinamide ring of NADP+ and the carbonyl oxygen of the inhibitors was consistent with the notion that MCFLA was closer to NADP+ $(\sim 6.57 \AA)$ than MPA $(\sim 7.68 \AA)$. These results indicate that MPA is the best selective inhibitor of AKR1C3, while MCFLA partially interacts with the NADP+ binding site, showing a relatively poor selectivity.

\section{Discussion}

It has been reported that AKR1C enzymes are overexpressed in several cancer cell types, such as bladder cancer cells and gastric cancer cells, contributing to the resistance to Cis-platin treatment[2-6,11]. Some cytotoxic lipid peroxidative products have been mentioned as targets of AKR1Cs that are involved in Cis-platin resistance[6]. However, the role of AKR1Cs in the mechanism underlying Cis-platin resistance remains unclear. We found that AKR1C inhibitors could reverse resistance of HepG2-RC cells, even though AKR1Cs were not significantly upregulated in HepG2-RC cells. The effects of siAKR1Cs on HepG2-RC cells were comparable to 
those of the inhibitor MPA, but could only partially explain the effects of MCFLA. This fact could be explained by that MPA is a steroid analog, which shows a high selectivity, while MCFLA inhibits not only AKR1C enzymes but also other AKR enzymes. MCFLA belongs to the class of non-steroid anti-inflammatory inhibitors, which have been reported to also inhibit cyclooxygenases besides AKR1Cs, indicating its poor selectivity[12]. As shown in Fig. 4D, all AKR enzymes share a common set of residues to bind $\mathrm{NADP}(\mathrm{H})$. As shown in Fig. 4B, MCFLA preferred the oxygen site rather than the steroid pocket, indicating that MCFLA inhibits AKR1C3 by disturbing $\mathrm{NAD}(\mathrm{P})(\mathrm{H})$ binding.

These results indicate that MCFLA could inhibit some other AKR enzymes besides AKR1Cs. This could explain why MCFLA causes a stronger reversal of Cis-platin resistance of HepG2-RC cells than MPA. It is likely that $\mathrm{NAD}(\mathrm{P}) \mathrm{H}$ plays a key role here because it is a reducing force and a product of $\mathrm{NAD}(\mathrm{P}) \mathrm{H}$-dependent oxidoreductases. Reprogramming energy metabolism is considered as a hallmark of cancer cells in which $\mathrm{NAD}(\mathrm{P})$ or $\mathrm{NAD}(\mathrm{P}) \mathrm{H}$ levels are increased[13-15]. It has been reported that some $\mathrm{NAD}(\mathrm{P}) \mathrm{H}$-dependent oxidoreductases, such as ALDHs, increase $\mathrm{NAD}(\mathrm{P}) \mathrm{H}$ levels in the cytosol of cancer cells, which then serves as an electron source[16].

We also found that the amount of total NAD (NAD++NADH) in both HepG2 and HepG2-RC cells was approximately four-fold higher than in HL-7702 cells. Moreover, the ratio of NADH/NAD+ in HepG2-RC cells was increased as much as seven-fold compared to HepG2 cells. The increased ratio could be explained by two steps (Fig. 5). First, NADH is produced continuously in hepatic cancer cells. This is in contrast to the situation in normal cells, where AKRs function as reductases[1]. However, the continuously biosynthesized NAD in hepatic cancer cells would make $\mathrm{NAD}(\mathrm{P}) \mathrm{H}$-dependent enzymes catalyzing reactions in one direction, from $\mathrm{NAD}(\mathrm{P})$ to $\mathrm{NAD}(\mathrm{P}) \mathrm{H}$. Second, the produced $\mathrm{NAD}(\mathrm{P}) \mathrm{H}$ would deal with Cis-platin-induced peroxidative products directly or indirectly. Usually, NADPH maintains glutathione at the reduced state, which could detoxify reactive oxygen species. However, this could not alter the $\mathrm{NAD}(\mathrm{P}) \mathrm{H} / \mathrm{NAD}(\mathrm{P})$ ratio too much, because each reaction is reversible. It has also been reported that no alteration of resistance to Cis-platin or oxaliplatin occurred after GSH depletion in oxaliplatin-resistant human gastric adenocarcinoma TSGH cells[17], indicating GSH is not involved in the Cis-platin resistance.

Besides GSH, NAD(P)H could also react rapidly with moderately oxidizing radicals to repair biomolecules[18]. So, $\mathrm{NAD}(\mathrm{P}) \mathrm{H}$ could also work as a directly operating antioxidant that scavenges radicals as $\mathrm{NAD}(\mathrm{P}) \mathrm{H}^{*}$ forms. These forms would exist in the cell for a relatively long time and keep the intracellular concentration of free $\mathrm{NAD}(\mathrm{P}) \mathrm{H}$ very low. Therefore, $\mathrm{NAD}(\mathrm{P}) \mathrm{H}$-dependent enzymes could catalyze $\mathrm{NAD}(\mathrm{P})$ to $\mathrm{NAD}(\mathrm{P}) \mathrm{H}$ continuously, resulting in a high ratio of $\mathrm{NAD}(\mathrm{P}) \mathrm{H} / \mathrm{NAD}(\mathrm{P})$. If these enzymes were widely inhibited by a poor selective inhibitor, such as MCFLA, NADH would not accumulate anymore, and Cis-platin resistance would be suppressed as well. 


\section{Conclusions}

271 In summary, it is believed that $\mathrm{NAD}(\mathrm{P}) \mathrm{H}$-dependent oxidoreductases, especially

272 AKRs, produce NADH in HepG2 cells to overcome Cis-platin-induced cytotoxicity.

273 According to this notion, chemotherapy with inhibitors, which could compete with

$274 \mathrm{NAD}(\mathrm{P})$ in most oxidoreductases, could lead to a better reversal of Cis-platin

275 resistance in Cis-platin-resistant cancer cells.

\section{$276 \quad 6 \quad$ Conflict of Interest}

277 The authors declare that the research was conducted in the absence of any commercial

278 or financial relationships that could be construed as a potential conflict of interest.

\section{$279 \quad 7 \quad$ Author Contributions}

280 Yuanhua Yu conceived-designed experiment.Tingting Sun and Le Gao analyzed data

281 and wrote and edited the main manuscript text. Xin Wang, Xue Sun and Rui Guo

282 performed in vitro experiments. All authors read and approval of the manuscript.

\section{$283 \quad 8 \quad$ Funding}

284 Supported by the grant provided by Science and Technology Development Plan

285 Project of Jilin Province, China(20200708101YY), Bioassay Engineering Technology

286 Application Technology Innovation Center of Jilin Province, China(20190907004TC).

\section{Acknowledgments}

288 We thank LetPub (www.letpub.com) for its linguistic assistance during the preparation 289 of this manuscript.

\section{Supplementary Material}

Additional tables(Table.S1., Table.S2., Table.S3.)

\section{Reference}

[1] Penning, T. M. The aldo-keto reductases (AKRs): Overview. Chemico-Biological Interactions. (2015); 234, 236-246. https://doi.org/10.1016/j.cbi.2014.09.024 aldo-keto reductase family protein AKR1B10 is highly correlated with smokers' non-small cell lung carcinomas. Clinical Cancer Research. (2005); 11(5), 1776-1785. https://doi.org/10.1158/1078-0432.CCR-04-1238

299 [3] Byrns, M. C., Mindnich, R., Duan, L., et al. Overexpression of aldo-keto reductase

300 1C3 (AKR1C3) in LNCaP cells diverts androgen metabolism towards testosterone

301 resulting in resistance to the $5 \alpha$-reductase inhibitor finasteride. Journal of Steroid

302 Biochemistry and Molecular Biology. (2012); 130(1-2), 7-15.

303 https://doi.org/10.1016/j.jsbmb.2011.12.012 
304

305

306

307

308

309

310

311

312

313

314

315

316

317

318

319

320

321

322

323

324

325

326

327

328

329

330

331

332

333

334

335

336

337

338

339

340

341

342

343

344

345

346

347

[4] Rižner, T. L., Šmuc, T., Rupreht, R., et al. AKR1C1 and AKR1C3 may determine progesterone and estrogen ratios in endometrial cancer. Molecular and Cellular Endocrinology. (2006); 248(1-2), 126-135. https://doi.org/10.1016/j.mce.2005.10.009 [5] Chen, C. C., Chu, C. B., Liu, K. J., et al. Gene expression profiling for analysis acquired oxaliplatin resistant factors in human gastric carcinoma TSGH-S3 cells: The role of IL-6 signaling and Nrf2/AKR1C axis identification. Biochemical Pharmacology. (2013); 86(7), 872-887. https://doi.org/10.1016/j.bcp.2013.07.025

[6] Matsumoto, R., Tsuda, M., Yoshida, K., et al. Aldo-keto reductase 1C1 induced by interleukin-1 $\beta$ mediates the invasive potential and drug resistance of metastatic bladder cancer cells. Scientific Reports. (2016); 6. https://doi.org/10.1038/srep34625

[7] Bellmunt, J., Albiol, S., Suárez, C., et al. Optimizing therapeutic strategies in advanced bladder cancer: Update on chemotherapy and the role of targeted agents. Critical Reviews in Oncology/Hematology. (2009); 69(3), 211-222. https://doi.org/10.1016/j.critrevonc.2008.06.002

[8] Li, Z., Yan, X., Sun, Y., et al. Expression of ADP-ribosyltransferase 1 is associated with poor prognosis of glioma patients. Tohoku Journal of Experimental Medicine. (2016); 239(4), 269-278. https://doi.org/10.1620/tjem.239.269

[9] Song, G. L., Jin, C. C., Zhao, W., et al. Regulation of the RhoA/ROCK/AKT/catenin pathway by arginine-specific ADP-ribosytransferases 1 promotes migration and epithelial-mesenchymal transition in colon carcinoma. International Journal of Oncology. (2016); 49(2), 646-656. https://doi.org/10.3892/ijo.2016.3539

[10]Yang, L., Xiao, M., Li, X., et al. Arginine ADP-ribosyltransferase 1 promotes angiogenesis in colorectal cancer via the PI3K/Akt pathway. International Journal of Molecular Medicine. (2016); 37(3), 734-742. https://doi.org/10.3892/ijmm.2016.2473

[11]Shirato, A., Kikugawa, T., Miura, N., et al. Cisplatin resistance by induction of aldo-keto reductase family 1 member $\mathrm{C} 2$ in human bladder cancer cells. Oncology Letters. (2014); 7(3), 674-678. https://doi.org/10.3892/ol.2013.1768

[12]Brozic, P., Turk, S., Lanisnik Rizner, T., et al. Inhibitors of Aldo-Keto Reductases AKR1C1-AKR1C4. Current Medicinal Chemistry. (2011); 18(17), 2554-2565. https://doi.org/10.2174/092986711795933713

[13]Roizen, M. F. Hallmarks of Cancer: The Next Generation. Yearbook of $\begin{array}{lllll}\text { Anesthesiology and Pain } & \text { Management. }\end{array}$ https://doi.org/10.1016/j.yane.2012.02.046

[14]GAO, P., \& WEI, H. Regulation of cancer cell metabolism. SCIENTIA SINICA Vitae. (2017); 47(1), 132-139. https://doi.org/10.1360/n052016-00334

[15]Mayevsky, A. Mitochondrial function and energy metabolism in cancer cells: Past overview and future perspectives. Mitochondrion. (2009); 9(3), 165-179. https://doi.org/10.1016/j.mito.2009.01.009

[16]Kim, S. Y. Cancer energy metabolism: Shutting power off cancer factory. Biomolecules and Therapeutics. (2018); 26(1), 39-44. https://doi.org/10.4062/biomolther.2017.184

[17]Chen, C. C., Chen, L. T., Tsou, T. C., et al. Combined modalities of resistance in an oxaliplatin-resistant human gastric cancer cell line with enhanced sensitivity to 
5-fluorouracil. British Journal of Cancer. (2007); 97(3), 334-344. https://doi.org/10.1038/sj.bjc.6603866 [18] Kirsch, M., \& Groot, H. NAD(P)H, a directly operating antioxidant? The FASEB Journal. (2001); 15(9), 1569-1574. https://doi.org/10.1096/fj.00-0823hyp

\section{Figure Legends}

Fig. 1. Differential expression of AKR1Cs in hepatic cell lines HL-7702, HepG2, and HepG2-RC, as measured with western blot. A, AKR1C1; B, AKR1C2; C, AKR1C3; $\mathrm{D}, \mathrm{AKR} 1 \mathrm{C} 4$. $\beta$-actin was selected as an internal reference. The signal intensity of each band was assessed with ImageJ software and divided by the value of $\beta$-actin. The ratios of AKR1Cs and $\beta$-actin are shown at the top of the figure.

Fig. 2. Reversion of Cis-platin sensitivity in HepG2-RC. A, IC50 value of three inhibitors acting on AKR1C3. MPA, medroxyprogesterone acetate; MCFLA, meclofenamic acid; MLS, methyliasmonate. B, Reversion ratio of Cis-platin sensitivity in HepG2-RC cells caused by three inhibitors. C, Knockdown of AKR1Cs by siRNA. Successful knockdown was confirmed with western blot. D, Reversion of Cis-platin sensitivity in AKR1C knockdown cells.

Fig. 3. Ratio of NADH/NAD+ in HL-7702, HepG2, and HepG2-RC cells. The amounts of total (NAD++NADH) and NADH were measured with an NAD/NADH quantitation kit. The amount of NAD was calculated from these two values. Finally, a ratio of NADH vs NAD was obtained.

Fig. 4. Molecular docking simulation results of AKR1C3 and three inhibitors. A, Meclofenamic acid (MCFLA) with AKR1C3 alone or AKR1C3 and NADP+. B, Methyliasmonate (MLS) with AKR1C3 alone or AKR1C3 and NADP+. C, Medroxyprogesterone acetate (MPA) with AKR1C3 alone or AKR1C3 and NADP+. OS, oxygen site; SP, steroid-binding pocket. Distance was measured between the nitrogen atom of the nicotinamide ring and the carbonyl oxygen of the inhibitor. AKR1C3 is represented in cartoon style. NADP+ is shown as a stick model, and Tyr-55 and His-117 are shown as a stick-ball model. NADP+, Tyr-55, and His-117 contain an oxygen site (OS). The steroid pockets (SPs) are displayed in surface style. $\mathrm{D}$, The alignment of representative members of the AKR enzyme family (AKR1A1, AKR1B1, AKR1C3, AKR1D1, AKR1E2, and AKR7A2). The residues involved in the $\mathrm{NADP}+$ binding are placed in the box.

Fig. 5. A possible role of $\mathrm{NAD}(\mathrm{P}) \mathrm{H} / \mathrm{NAD}(\mathrm{P})$-dependent reductase/oxidase in the mechanism underlying Cis-platin resistance. Arrows pointing upwards indicate upregulation in HepG2-RC cells; arrows pointing downwards indicate downregulation in HepG2-RC cells.

Table.1. The mRNA amount (2- $\Delta \mathrm{Ct})$ of AKR1Cs in different hepatic cell lines measured by qRT-PCR. 


\begin{tabular}{cccc}
\hline Cell lines & HL-7702 & HepG2 & HepG2-RC \\
\hline AKR1C1 & 10.3 & $3.1 \times 10^{3}$ & $0.3 \times 10^{3}$ \\
AKR1C2 & 6.8 & $0.3 \times 10^{3}$ & $0.1 \times 10^{3}$ \\
AKR1C3 & 16.1 & $6.1 \times 10^{4}$ & $2.9 \times 10^{6}$ \\
AKR1C4 & 4.6 & 6.6 & 1.6 \\
\hline
\end{tabular}

386 Table.2. Regulation of AKR enzymes measured by RNA-seq.

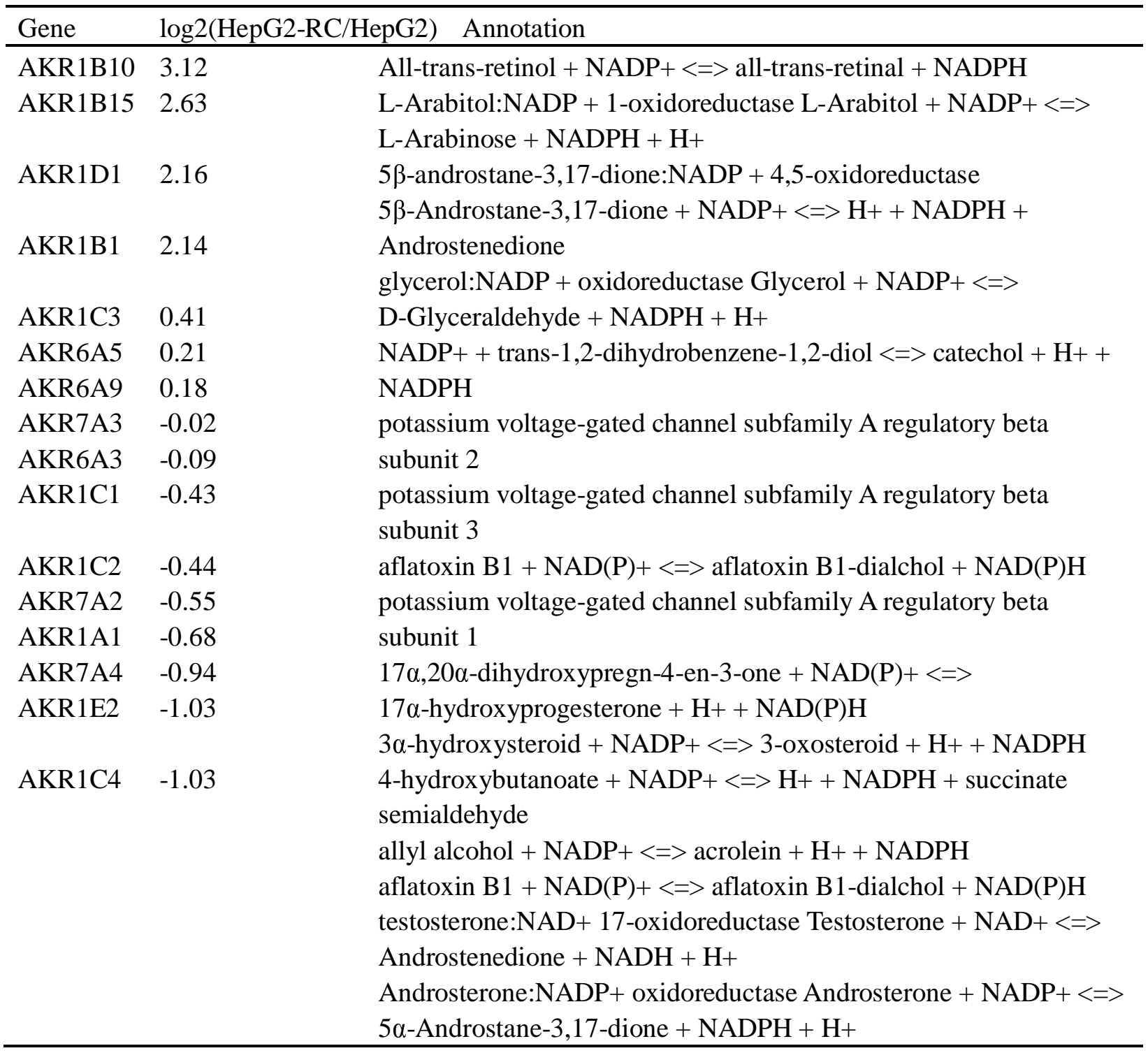

387 Table.3. Regulation of NAD metabolism related genes in HepG2 and HepG2-RC

388 measured by RNA-seq. 


\begin{tabular}{|c|c|c|}
\hline Gene & \multicolumn{2}{|c|}{ Log2(HepG2-RC/HepG2) Annotation } \\
\hline IDO1 & 0.41 & indoleamine 2,3-dioxygenase 1 \\
\hline IDO2 & NA & indoleamine 2,3-dioxygenase 2 \\
\hline TDO2 & 3.97 & tryptophan 2,3-dioxygenase \\
\hline KMO & -0.42 & kynurenine 3-monooxygenase \\
\hline KYNU & 2.78 & kynureninase \\
\hline HAAO & -0.03 & 3-hydroxyanthranilate 3,4-dioxygenase \\
\hline QPRT & 0.076 & quinolinate phosphoribosyltransferase \\
\hline NMRK1 & 0.33 & nicotinamide riboside kinase 1 \\
\hline NMRK2 & NA & nicotinamide riboside kinase 2 \\
\hline NMNAT1 & 0.03 & nicotinamide nucleotide adenylyltransferase 1 \\
\hline NMNAT2 & 3.76 & nicotinamide nucleotide adenylyltransferase 2 \\
\hline NMNAT3 & -0.10 & nicotinamide nucleotide adenylyltransferase 3 \\
\hline Naprt & -0.71 & nicotinate phosphoribosyltransferase \\
\hline NAMPT & 0.83 & nicotinamide phosphoribosyltransferase \\
\hline Nadsyn 1 & 0.4 & NAD synthetase 1 \\
\hline SIRT1 & -0.16 & sirtuin 1 \\
\hline SIRT2 & 0.30 & sirtuin 2 \\
\hline SIRT3 & -0.61 & sirtuin 3 \\
\hline SIRT4 & -0.012 & sirtuin 4 \\
\hline SIRT5 & -0.23 & sirtuin 5 \\
\hline SIRT6 & -0.11 & sirtuin 6 \\
\hline SIRT7 & 0.27 & sirtuin 7 \\
\hline CD38 & -1.71 & CD38 molecule \\
\hline PARP1 & -0.20 & poly(ADP-ribose) polymerase 1 \\
\hline PARP2 & -0.68 & poly(ADP-ribose) polymerase 2 \\
\hline PARP3 & 0.04 & poly(ADP-ribose) polymerase 3 \\
\hline PARP4 & 0.48 & poly(ADP-ribose) polymerase 4 \\
\hline PARP6 & -0.33 & poly(ADP-ribose) polymerase 6 \\
\hline PARP8 & 0.22 & poly(ADP-ribose) polymerase 8 \\
\hline PARP9 & 1.64 & poly(ADP-ribose) polymerase 9 \\
\hline PARP10 & 1.1 & poly(ADP-ribose) polymerase 10 \\
\hline PARP11 & NA & poly(ADP-ribose) polymerase 11 \\
\hline PARP12 & 1.14 & poly(ADP-ribose) polymerase 12 \\
\hline PARP14 & 1.22 & poly(ADP-ribose) polymerase 13 \\
\hline PARP15 & 0.96 & poly(ADP-ribose) polymerase 15 \\
\hline PARP16 & -0.49 & poly(ADP-ribose) polymerase 16 \\
\hline ART1 & 4.06 & ADP-ribosyltransferase 1 \\
\hline ART3 & 1.24 & ADP-ribosyltransferase 3 \\
\hline ART4 & 1.29 & ADP-ribosyltransferase 4 \\
\hline ART5 & 3.73 & ADP-ribosyltransferase 5 \\
\hline
\end{tabular}


Fig 1

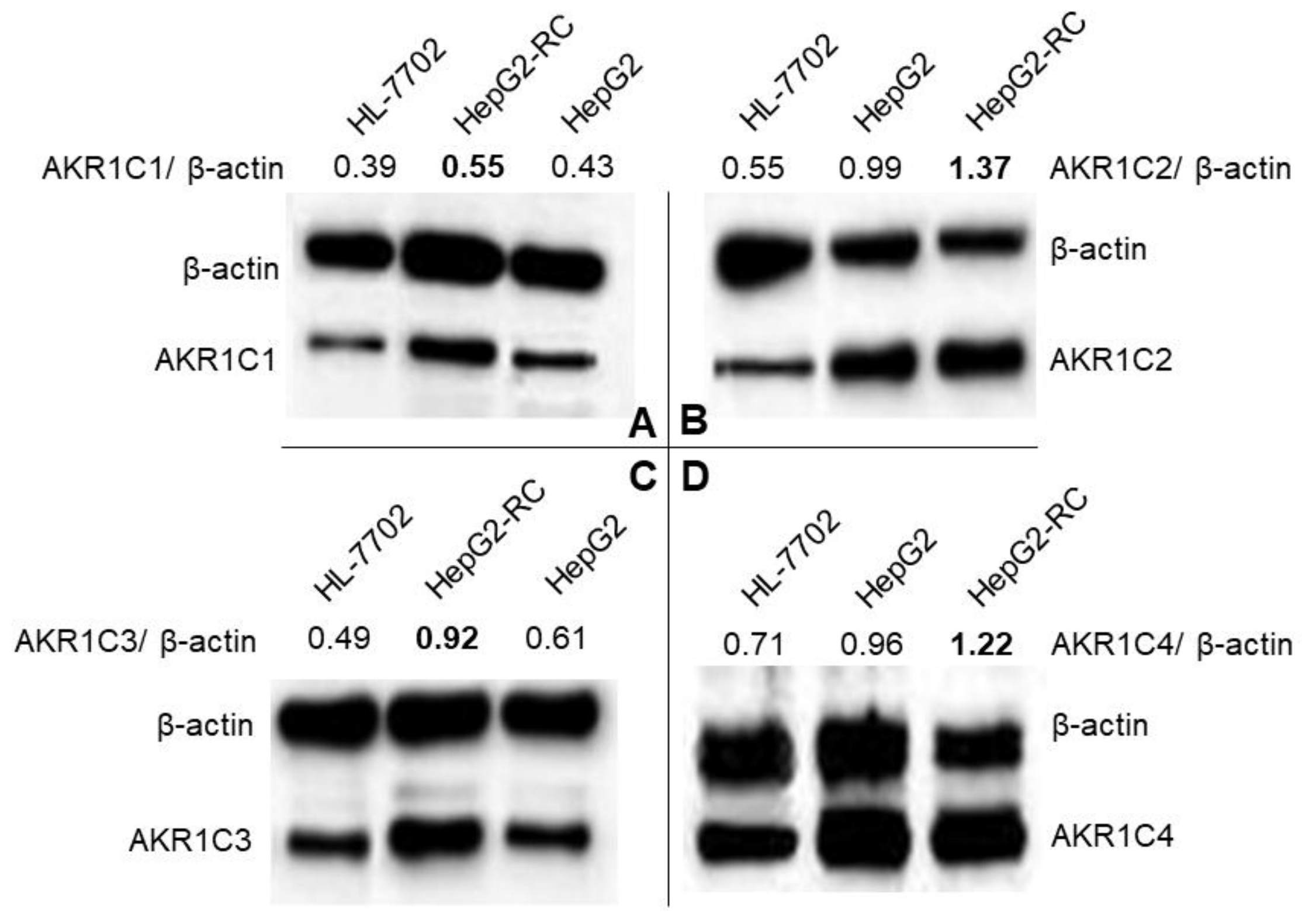




\section{Fig 3}

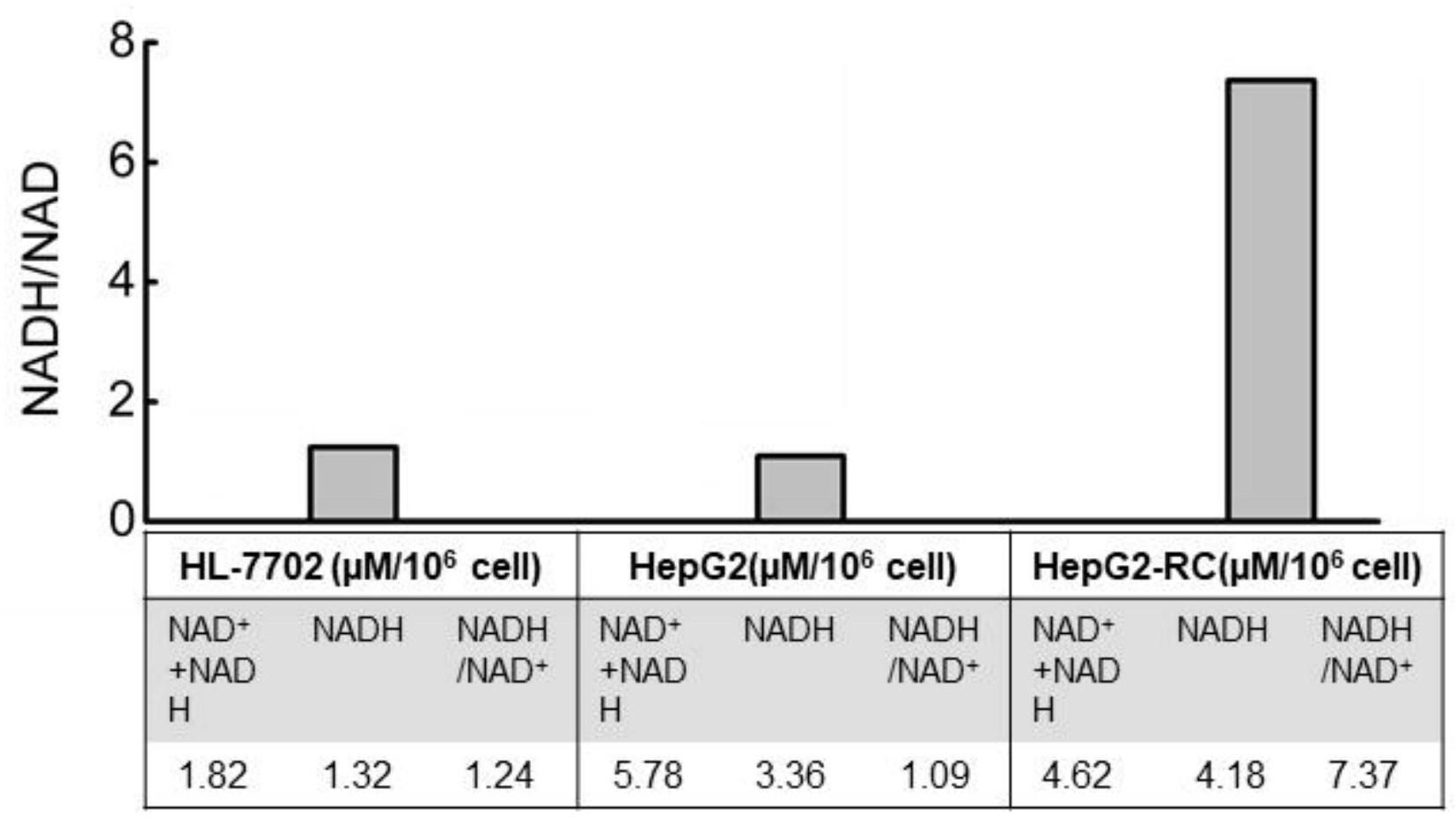


A B

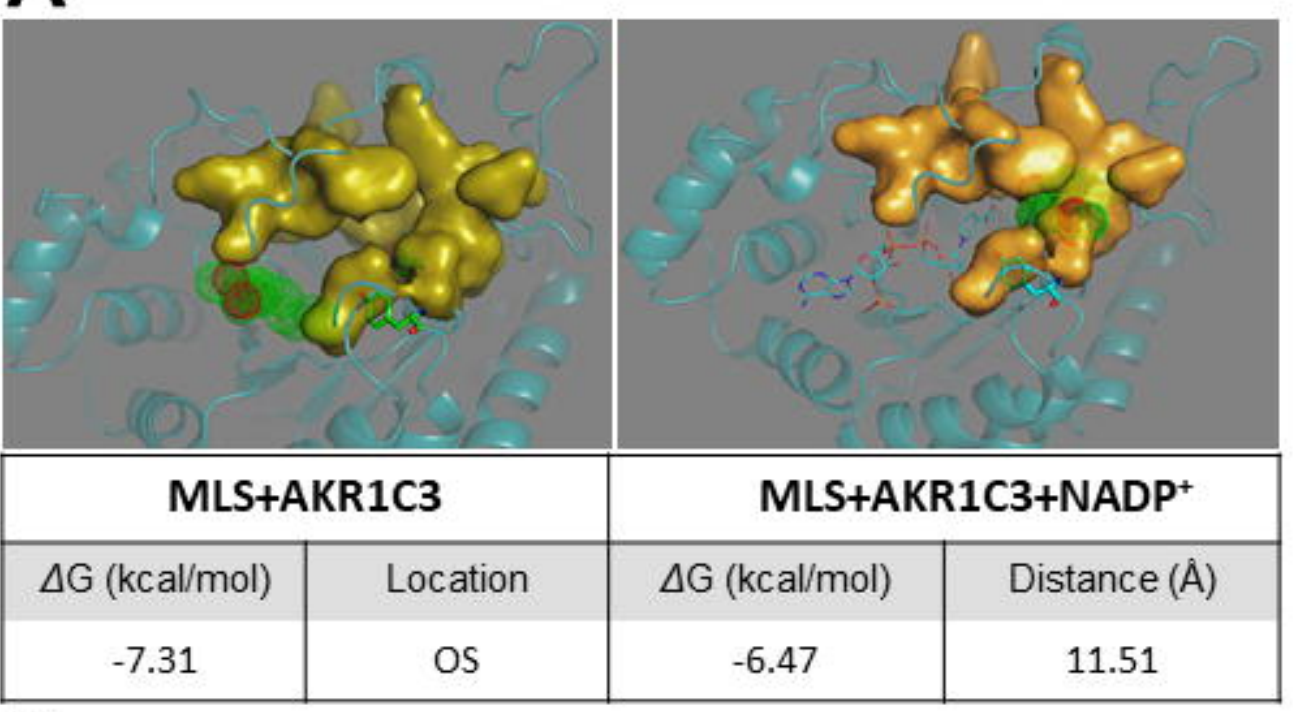

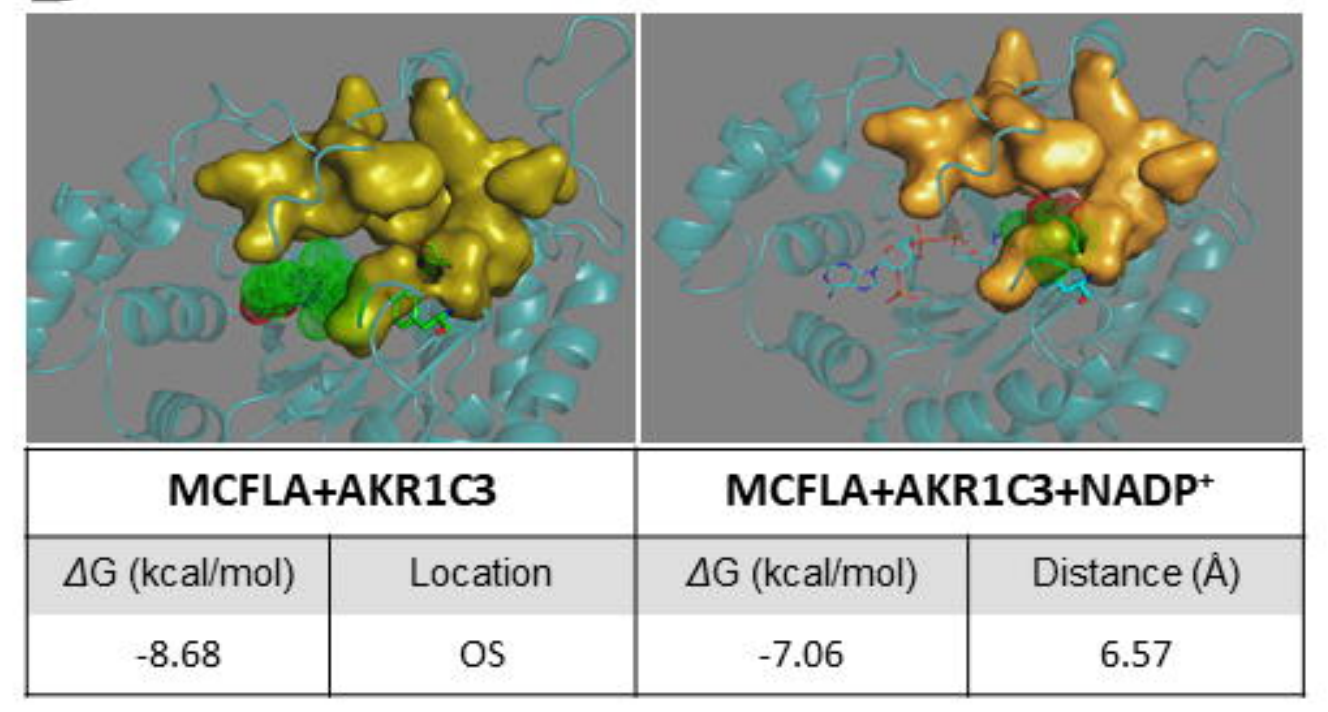

C

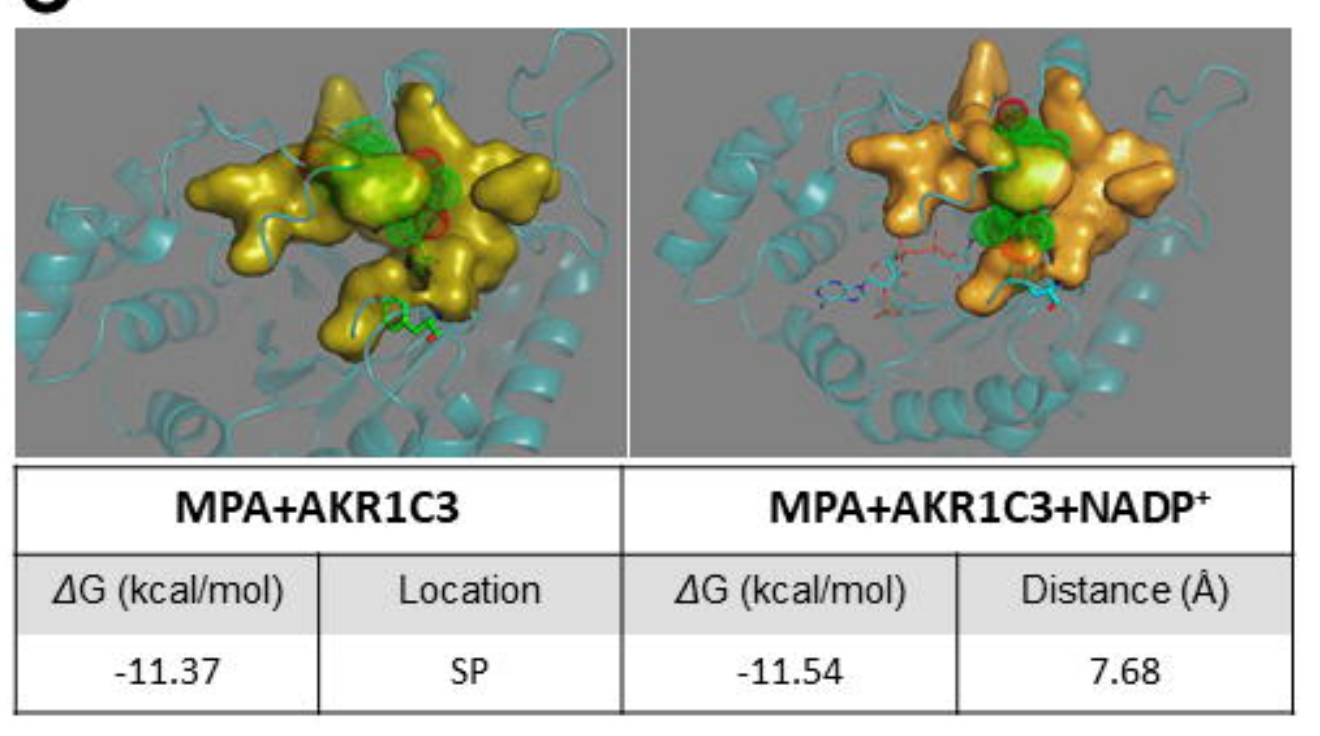

\section{D}

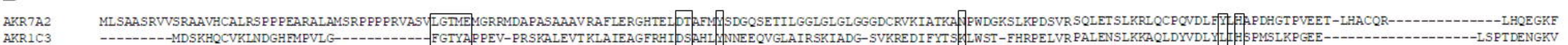

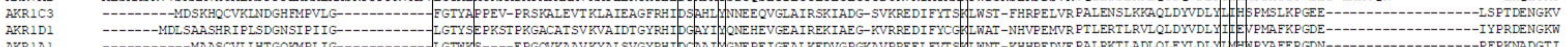

AKRIAI

ARRIBI

-

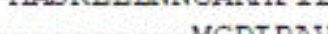

PFPKNADGII

AKR1E2

AKR7A2 2
AKR1C3

ARRIC3

AKRIDI

AKR1B1
AKR1E2

AKR7A2
AKR1C3

AKRIDI

ARRIAI SSWZS-OVELGLSNYASWEVAEICTICKSNGWILPTVYQGMRNATTRQVET-------1. IYHKSNLCATWEAMEACKDA---GLVR---SLGVENENRRQLELILNKPGLKHKFVSNFV ECHFYFTQPKLIKFCQQHDIVITAYSPLGT CYDSTHYKETWKALEALVAK---GLVQ---ALGL N N N NSROIDDILSVAS--VRPAVI DV ECHPYLAQNELIAHCQARGLEVIAYSPLGS VPSDTNILDTWAAMEELVDE---GLVK---AIGI FN FNHLVVEMILNKPGLKYKPAVN PI ECHPYLTQEKLIQYCQSKGIVVIAYSPLGS IPSDTDFLDTWEAMEDLVIT---GLVK---NI GVENENHEQLERLLNKP GLRFKPLTN PI ECHPYLTQRNLISFCQSRDVSVIAYRPLGG.

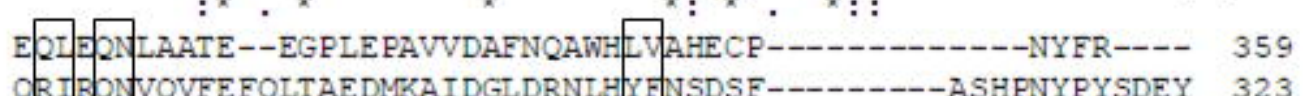
DRARRDDEPV HEEPV LALAEKYGR PP-- AOIIE RWQVQR-------KVIDI I PKSIIP

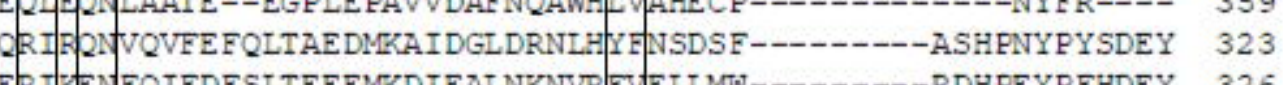
E

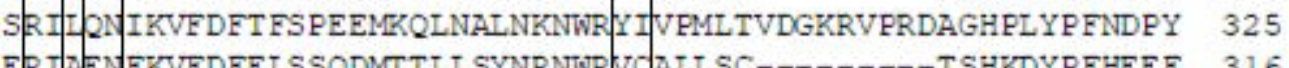

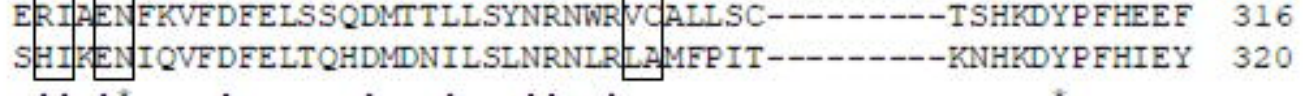


Fig 5

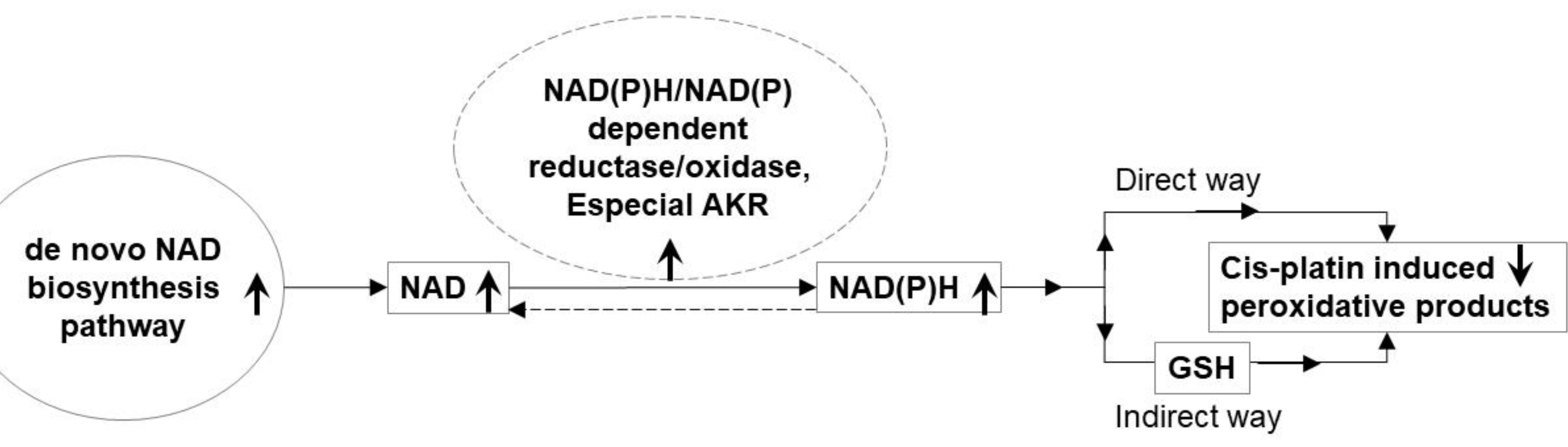


Fig 2

A

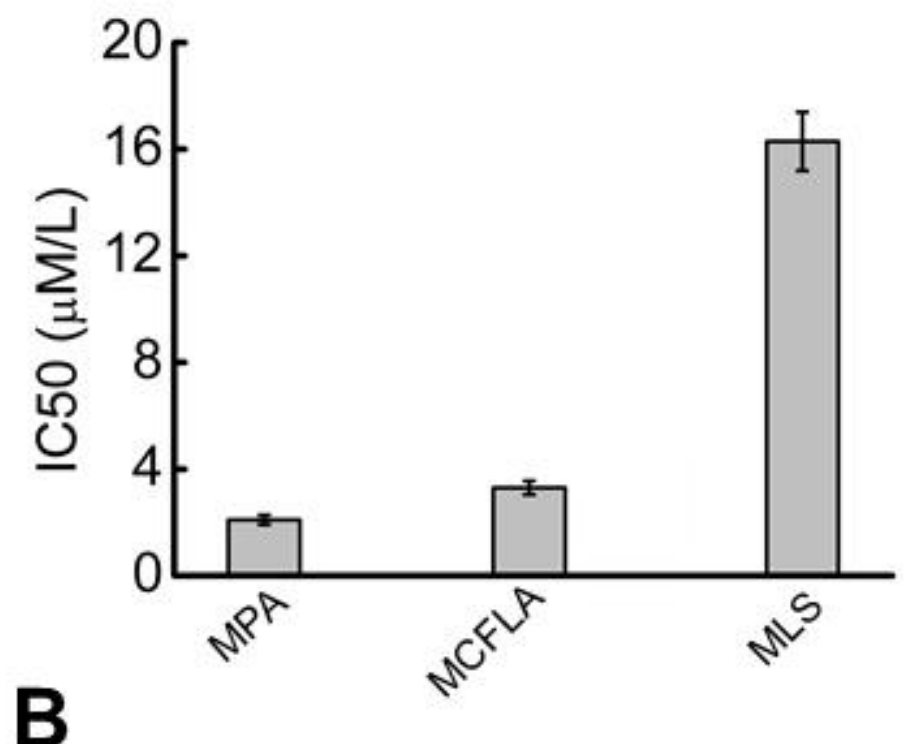

B

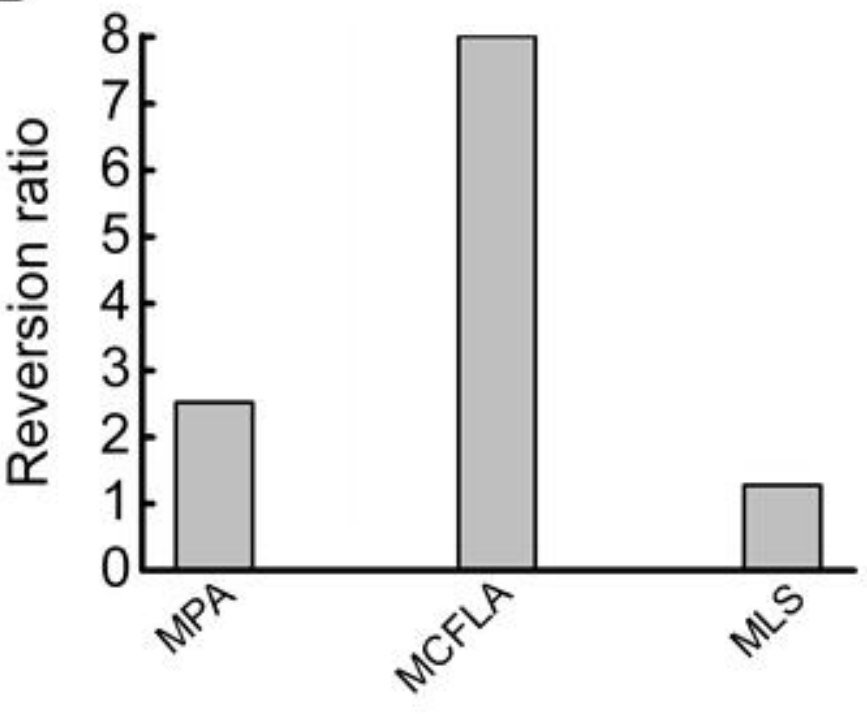

C

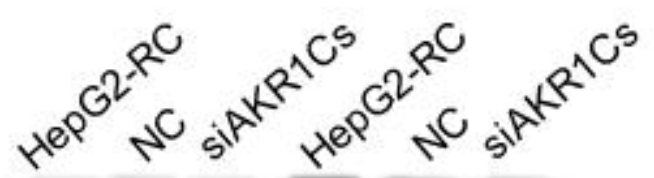

$\beta$-actin AKR1C1

$\beta$-actin

AKR1C3

-actin

D

\begin{tabular}{|lcc|}
\hline \multicolumn{3}{|l|}{ Reversion of Cis-platin sensitivity in } \\
AKR1Cs knock-down cell \\
\hline HepG2-RC & HepG2-RC+siRNA & Ration \\
\hline $1.5 \mu \mathrm{g} / \mathrm{ml}$ & $0.74 \mu \mathrm{g} / \mathrm{ml}$ & 2.04 \\
\hline
\end{tabular}

Reversion of Cis-platin sensitivity in AKR1Cs knock-down cell 